\title{
Terminal spreading depolarizations causing electrocortical silencing prior to clinical brain death: case report
}

\author{
Andrew P. Carlson, MD, MSCR, ${ }^{1}$ C. William Shuttleworth, PhD, ${ }^{2}$ Sebastian Major, MD, ${ }^{3-5}$ \\ Coline L. Lemale, ${ }^{3,4}$ Jens P. Dreier, MD, ${ }^{3-7}$ and Jed A. Hartings, PhD ${ }^{8}$ \\ Departments of ${ }^{1}$ Neurosurgery and ${ }^{2}$ Neurosciences, University of New Mexico, Albuquerque, New Mexico; ${ }^{3}$ Center for Stroke \\ Research Berlin, Charité-Universitätsmedizin Berlin, Corporate Member, Freie Universität Berlin, Humboldt-Universität zu \\ Berlin, and Berlin Institute of Health; ${ }^{4}$ Department of Experimental Neurology, Charité-Universitätsmedizin Berlin, Corporate \\ Member, Freie Universität Berlin, Humboldt-Universität zu Berlin, and Berlin Institute of Health; ${ }^{5}$ Department of Neurology, \\ Charité-Universitätsmedizin Berlin, Corporate Member, Freie Universität Berlin, Humboldt-Universität zu Berlin, and Berlin \\ Institute of Health; ${ }^{6}$ Bernstein Center for Computational Neuroscience Berlin; ${ }^{7}$ Einstein Center for Neurosciences Berlin, \\ Germany; and ${ }^{8}$ Department of Neurosurgery, University of Cincinnati, Ohio
}

\begin{abstract}
The authors report on a 57-year-old woman in whom progression to brain death occurred on day 9 after aneurysmal subarachnoid hemorrhage without evidence of significant brain edema or vasospasm. Neuromonitoring demonstrated that brain death was preceded by a series of cortical spreading depolarizations that occurred in association with progressive hypoxic episodes. The depolarizations induced final electrical silence in the cortex and ended with a terminal depolarization that persisted $>7$ hours. To the authors' knowledge, this is the first report of terminal spreading depolarization in the human brain prior to clinical brain death and major cardiopulmonary failure.
\end{abstract}

https://thejns.org/doi/abs/10.3171/2018.7.JNS181478

KEYWORDS subarachnoid hemorrhage; brain death; electrophysiology; electrocorticography; spreading depolarization; vascular disorders

$\mathrm{S}$ PREADING depolarizations (SDs) are waves of mass tissue depolarization that occur in the cerebral cortex and are the hypothesized mechanism of acute lesion development in a variety of diseases and injury conditions. ${ }^{2,10}$ They mediate a toxic collapse of cellular and mitochondrial ion gradients, release of neurotransmitters, and development of cell swelling known as cytotoxic edema. SDs are recoverable and relatively brief (approximately 2 minutes) in the normal brain, but they become prolonged when the energy supply is locally compromised due to hypoxia/ischemia or inverse neurovascular coupling known as spreading ischemia. ${ }^{1}$ In such pathological conditions, depolarization beyond a threshold duration results in tissue necrosis. In patients, SDs are associated with the following: 1) worse outcomes after brain trauma; ${ }^{8}$ 2) both acute and delayed brain lesions after aneurysmal sub- arachnoid hemorrhage (aSAH), ${ }^{6,11}$ even in the absence of large-vessel vasospasm; ${ }^{19}$ and 3) infarct growth after ischemic stroke. ${ }^{15}$ In the dying process after withdrawal of lifesustaining therapies, a prolonged terminal SD is observed in the human brain, following cardiac arrest, the development of critical cerebral ischemia, and brain isoelectricity; ${ }^{4}$ however, this phenomenon has not been previously documented in the absence of circulatory collapse. Here, by contrast, we report a case of brain death developing on day 9 after aSAH in association with a series of SDs that occurred prior to cardiopulmonary collapse or brain isoelectricity. In this case, SDs contributed to development of brain isoelectricity and evolved into more prolonged and eventually terminal depolarization events, consistent with their mediation of electrophysiological and clinical brain death without cardiopulmonary failure.

ABBREVIATIONS aSAH = aneurysmal subarachnoid hemorrhage; $\mathrm{DC}=$ direct current; $\mathrm{ECoG}=$ electrocorticography; ICP = intracranial pressure; NUP = negative ultraslow potential; $\mathrm{p}_{\mathrm{ti}} \mathrm{O}_{2}=$ brain tissue partial pressure of oxygen; $\mathrm{SD}=$ spreading depolarization; $\mathrm{SpO}_{2}=$ peripheral capillary oxygen saturation.

SUBMITTED May 25, 2018. ACCEPTED July 16, 2018.

INCLUDE WHEN CITING Published online December 7, 2018; DOI: 10.3171/2018.7.JNS181478. 


\section{Case Report Methods}

Standard multimodal monitoring was performed at the University of New Mexico, and in addition, electrocorticography (ECoG) to monitor SDs was performed under an observational IRB-approved research protocol. The details of this monitoring protocol have been previously published under consensus recommendations of the COSBID (Cooperative Studies on Brain Injury Depolarizations) consortium. ${ }^{2}$ Briefly, ECoG activity was recorded using a single platinum subdural electrode strip (Integra) and a direct-current (DC) amplifier., ${ }^{2,9}$ The safety of this monitoring technique in SAH patients has previously been demonstrated. ${ }^{7}$ Other continuous physiological variables included blood pressure, heart rate, peripheral oxygen saturation, intracranial pressure (ICP), and brain tissue partial pressure of oxygen $\left(\mathrm{p}_{\mathrm{ti}} \mathrm{O}_{2}\right.$; Licox, Integra). Invasive parenchymal monitoring $\left(\mathrm{p}_{\mathrm{ti}} \mathrm{O}_{2}\right.$ and $\left.\mathrm{ICP}\right)$ was performed via a bolt-based neuromonitoring system on the right (contralateral to the side of surgery). The ECoG strip was placed at the time of surgery on the left frontal cortex and later removed at the bedside with gentle traction. All data were acquired using a Component Neuromonitoring System (Moberg ICU Solutions, Inc.) and exported to LabChart v8.1.3 (AD instruments) for analysis. ${ }^{2}$

\section{Case Study}

\section{Summary of Clinical Course}

This 57-year-old woman presented with sudden-onset headache and was diagnosed with aSAH (Hunt and Hess grade III, modified Fisher grade 4) from the rupture of a broad-neck anterior communicating artery aneurysm. Figure 1 presents the imaging studies and summary of the clinical course. The aneurysm was surgically clipped on day 2, and a subdural electrode strip was placed over the inferior frontal gyrus. Postoperatively, the patient followed commands in all 4 extremities and her examination status remained stable. She was extubated on day 3 but had respiratory difficulty and was reintubated the same day. Routine angiography on day 6 did not show any sign of vasospasm or residual aneurysm. While the patient recovered from anesthesia used for the angiogram, her motor status deteriorated to a motor score of withdrawal to pain only, and two SDs were observed shortly thereafter in ECoG recordings. A subsequent CT scan revealed a new medial frontal intracerebral hemorrhage thought to possibly be related to hemorrhagic conversion of a small ischemic infarct in the patient with permissive hypertension. ICP was moderately elevated at 20-30 mm Hg. The patient then developed renal dysfunction and was placed on continuous renal replacement therapy, and she further developed progressive bradycardia and hypotension requiring vasopressors, likely related to Takotsubo's cardiomyopathy. Despite these interventions, in the terminal hours on days 8 and 9, the patient experienced moderate hypoxia with peripheral capillary oxygen saturation $\left(\mathrm{SpO}_{2}\right)<90 \%$ and $\mathrm{p}_{\mathrm{ti}} \mathrm{O}_{2}$ decreasing to $<5 \mathrm{~mm} \mathrm{Hg}$. This coincided with a cluster of several SDs that were associated with persistent loss of cortical activity, as shown in Figs. 2-4. Shortly after these events, the patient was found to have an abrupt loss of brainstem reflexes and fixed dilated pupils. After discussion with the family regarding the poor prognosis, the patient was terminally extubated and expired shortly thereafter. The exact cause of death was unclear because there was no massive ICP increase suggestive of large stroke or edema and because cardiac, pulmonary, and renal function were thought to be adequately supported.

\section{Physiology With Progression to Electrical and Clinical Brain Death}

The full course of deterioration to brain death is shown in Fig. 2, and it began in conditions of impaired cerebral autoregulation. Decreases in heart rate and blood pressure resulted in coincident decreases in $\mathrm{SpO}_{2}$ and $\mathrm{p}_{\mathrm{ti}} \mathrm{O}_{2}$ that were initially moderate. There were then 3 severe cerebral hypoxic episodes, highlighted by arrows in Fig. 2 and shown in greater detail in Fig. 3, that triggered a sequence of terminal electrophysiological deterioration over the course of approximately 5 hours.

The first hypoxic episode in the 8th hour was a profound decrease of $\mathrm{p}_{\mathrm{ti}} \mathrm{O}_{2}$ to $5 \mathrm{~mm} \mathrm{Hg}$. Within minutes, this decrease was followed by an SD recorded on the ECoG strip in the hemisphere contralateral to $\mathrm{p}_{\mathrm{ti}} \mathrm{O}_{2}$ monitoring. After a transient recovery of $\mathrm{p}_{\mathrm{ti}} \mathrm{O}_{2}$ to $20 \mathrm{~mm} \mathrm{Hg}$ with blood pressure augmentation, a second drop in $\mathrm{SpO}_{2}$ and $\mathrm{p}_{\mathrm{ti}} \mathrm{O}_{2}$ then occurred about an hour later. This again was followed by an SD, which now induced a state of persistent electrical silence at electrodes 3-5. A final physiological derangement was noted again just over an hour later when ICP increased moderately to $30 \mathrm{~mm} \mathrm{Hg}$, resulting in a drop in cerebral perfusion pressure to $30 \mathrm{~mm} \mathrm{Hg}$ and $\mathrm{SpO}_{2}$ to $80 \%$. $\mathrm{P}_{\mathrm{ti}} \mathrm{O}_{2}$ also decreased to nearly zero and never recovered, despite restoration of $\mathrm{SpO}_{2}$ to $100 \%$. This $\mathrm{p}_{\mathrm{ti}} \mathrm{O}_{2}$ decrease developed simultaneously with a terminal SD at electrodes $1,2,4$, and 5 that then spread to electrode 6 after 5 minutes and then electrode 3 after 24 minutes. This SD induced persistent electrical silence at electrode 2 and near-complete silence at electrodes 1 and 6.

This final SD is shown in detail in Fig. 4 (arrows show onset). At all electrodes, this event was terminal, since the negative potential shift indicating tissue depolarization was persistent (7+ hours) or did not recover to baseline. This persistent potential shift, often of increasing amplitude, is described as a negative ultraslow potential (NUP). The NUP not only reflects cellular depolarization but also is thought to be generated in part by the progression from depolarization to cell death in an increasing fraction of cells. $^{3}$ A consequence of this cell death is that recovery from depolarization is only partial and that SDs subsequent to a prolonged NUP-generating event have reduced amplitude. ${ }^{14}$ This hypothesis is consistent with our observations of additional SDs with reduced amplitude superimposed on the NUP (Fig. 4), presumably occurring in the small fraction of viable cells. In electrodes 1 and 6 , these SDs completed the suppression of spontaneous activity to an isoelectric state.

\section{Discussion}

The cause of death in this case seems to have been a 

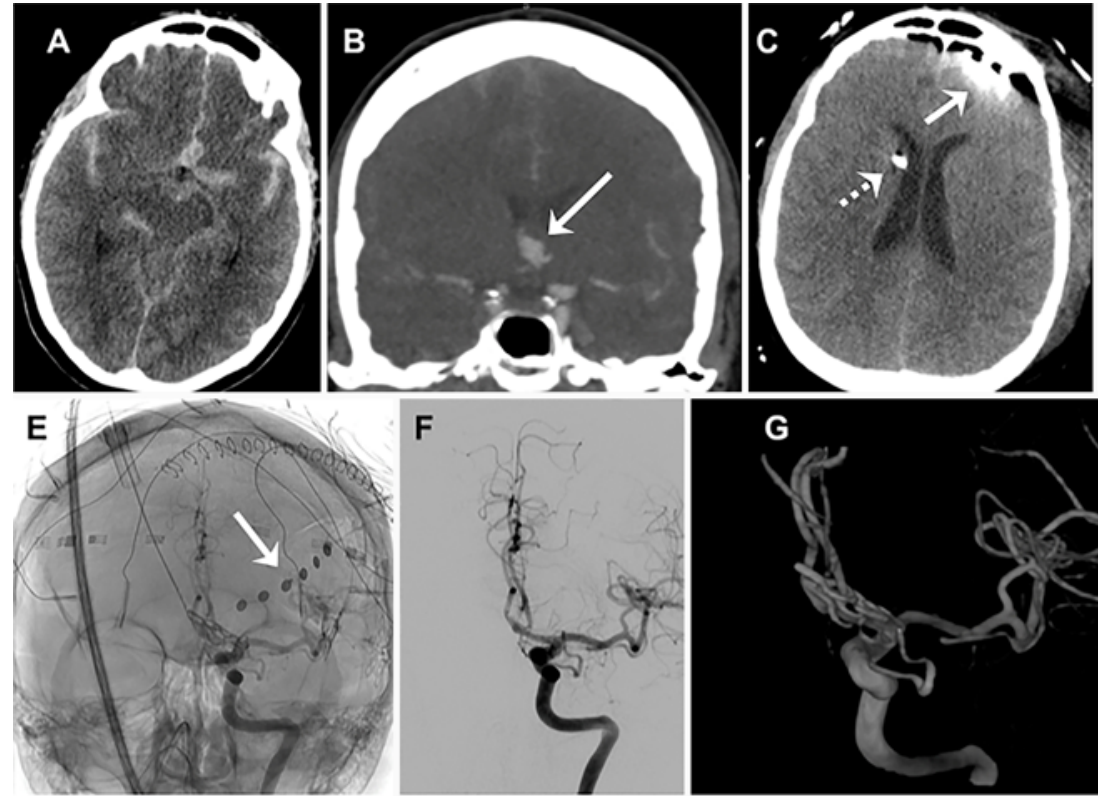

$\mathbf{F}$

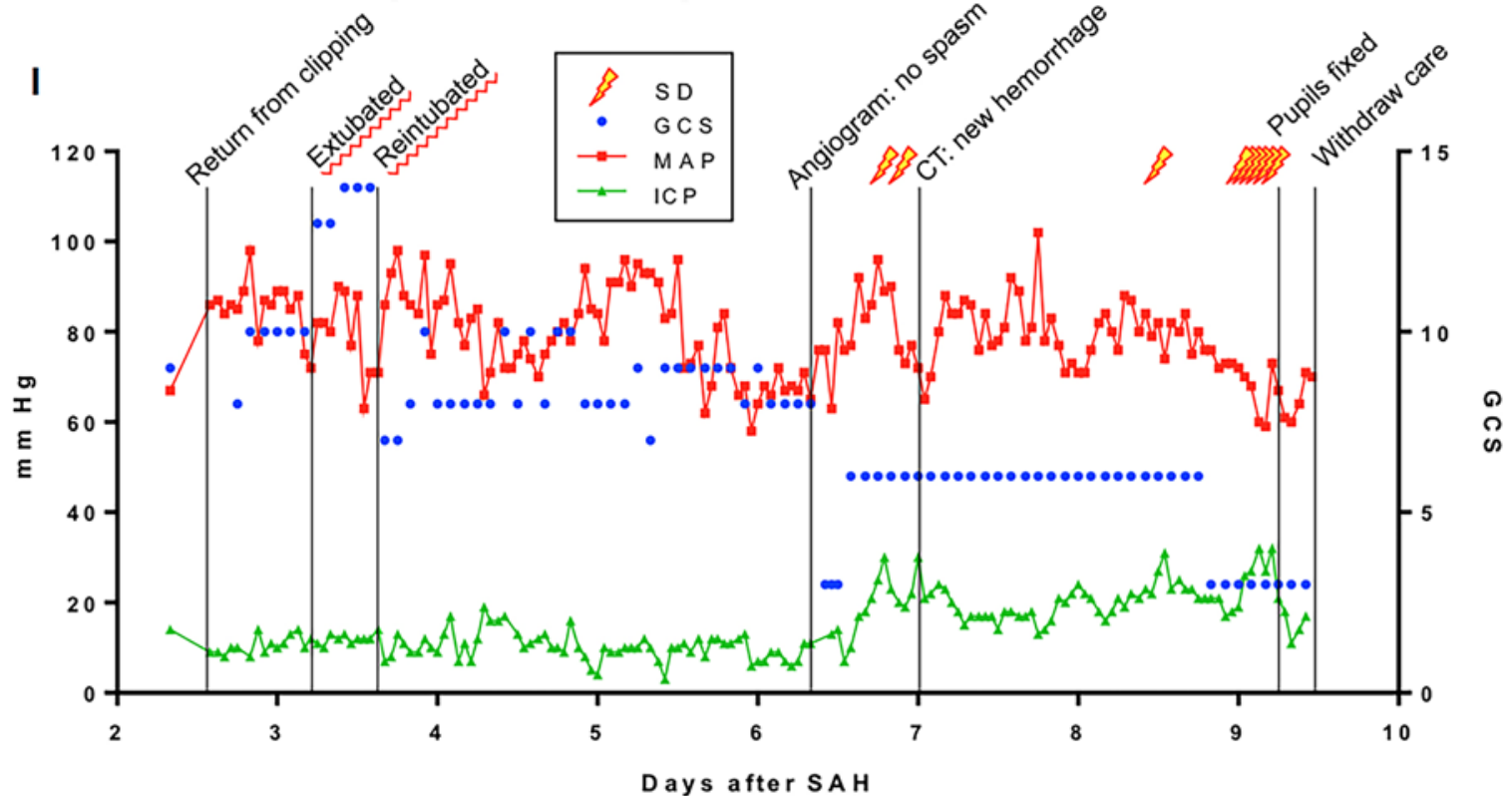

FIG. 1. Overview of imaging studies and clinical course. A: Initial axial CT scan from aSAH day 1, demonstrating thick subarachnoid hemorrhage in the basal cisterns, as well as interhemispheric and Sylvian fissures. B: Coronal reconstruction from a CT angiogram showing anterior communicating artery aneurysm (white arrow). C and D: Postoperative CT scans (aSAH day 3) showing the position of the electrode strip (solid arrow) and external ventricular drain (EVD) (dashed arrow) without evidence of large stroke or hemorrhage. E-G: Nonsubtracted angiogram, subtracted angiogram, and 3D reconstruction of left internal carotid artery angiogram demonstrating no significant angiographic vasospasm on $\mathrm{SAH}$ day 6 . The electrode strip $(\mathrm{E}$, arrow) can be visualized on the left frontal region. H: CT scan demonstrating a delayed left medial frontal intracerebral hematoma on aSAH day 6 . I: Time course of patient events, intracranial monitoring, neurological examination, and SDs. The final period prior to withdrawal of care (days 8 and 9) is illustrated in detail in Figs. 2-4. GCS = Glasgow Coma Scale; ICH = intracerebral hematoma; MAP = mean arterial pressure. Figure is available in color online only.

severe global hypoxia/ischemia that developed progressively in the context of impaired autoregulation and repetitive SDs. This is evidenced in the $\mathrm{p}_{\mathrm{ti}} \mathrm{O}_{2}$ measures that decreased abruptly, with drops in $\mathrm{SpO}_{2}$, and likely triggered the simultaneously occurring SDs., ${ }^{4,18}$ For the last two of these events, $\mathrm{P}_{\mathrm{ti}} \mathrm{O}_{2}$ did not recover but instead de- creased in a cumulative, persistent manner to $<5 \mathrm{~mm} \mathrm{Hg}$. It is unknown why $\mathrm{p}_{\mathrm{ti}} \mathrm{O}_{2}$ did not recover, but speculatively the mechanism of spreading ischemia ${ }^{1,5}$ could account for these persistent changes if SDs occurred not only at the location of electrode strip monitoring but also more globally to include the contralateral location of $\mathrm{p}_{\mathrm{ti}} \mathrm{O}_{2}$ monitor- 


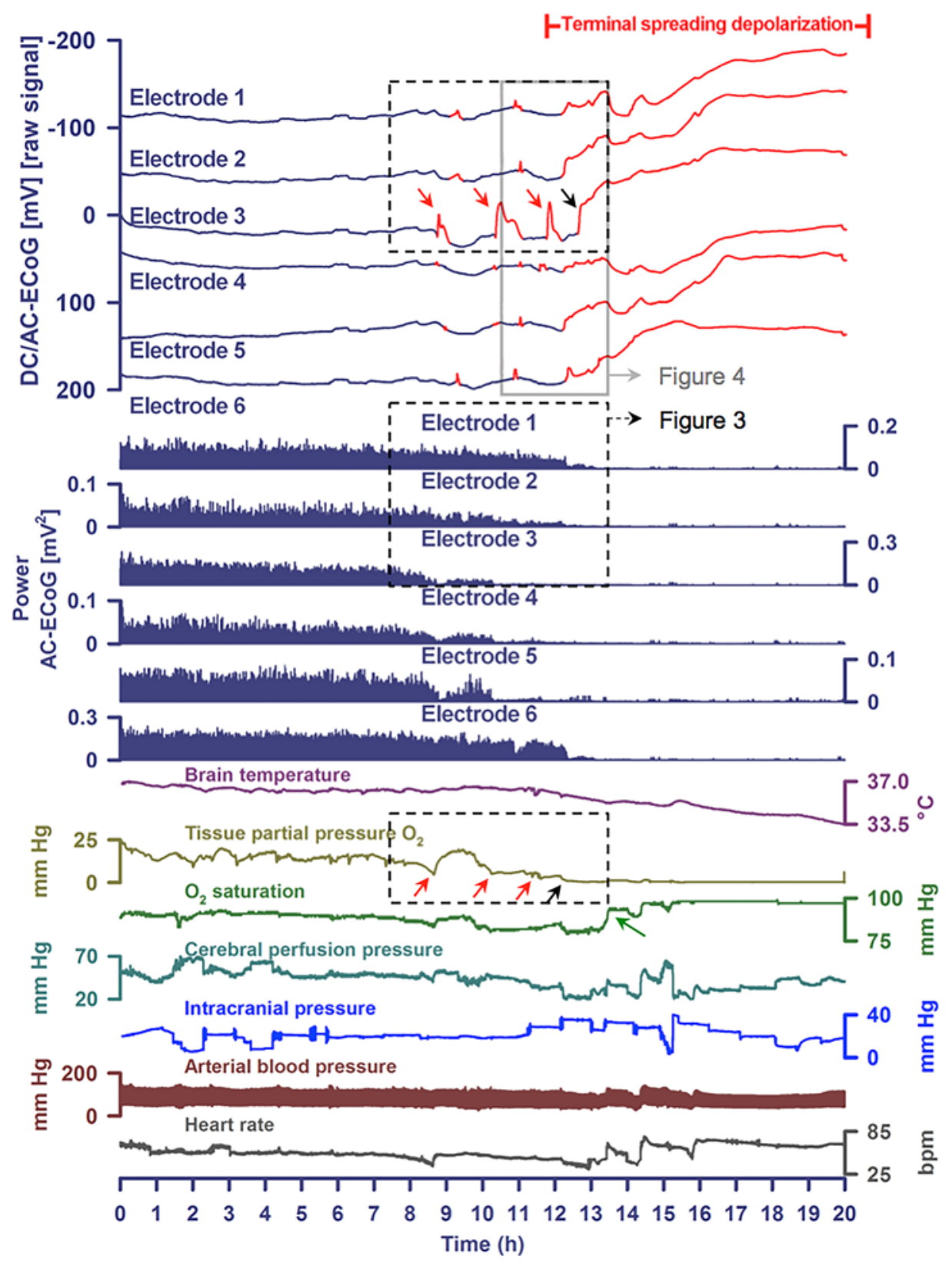

FIG. 2. Neuromonitoring during progression to brain death. Upper 6 traces show the raw DC recordings from each ECoG electrode (negative up). Red portions indicate potentials associated with SDs. These show a series of several transient SDs (best seen on electrode 3, red arrows) followed by terminal SD, which is manifested as a persistent negative potential shift (black arrow). Middle traces show the power of the spontaneous brain activity $(0.5-45.0 \mathrm{~Hz})$ recorded from the same 6 electrodes as shown above in the DC traces. ECoG power decreases progressively through the series of SDs until the entire electrode strip shows electrical silence. Lower 6 traces show associated continuous physiology variables over the same time period. The red arrows in the $\mathrm{p}_{\mathrm{ti}} \mathrm{O}_{2}$ (Licox) demonstrate time-linked episodes of brain hypoxia associated with the SDs measured from the contralateral hemisphere. There is progressive decrease to zero as the terminal depolarization occurs (black arrow), likely indicating a widespread wave of spreading ischemia. Though systemic oxygen saturation transiently dropped moderately with these events, the brain oxygenation did not recover despite systemic hyperoxygenation to $100 \%$ (green arrow). During this time, cerebral perfusion pressure dropped to the range of $30 \mathrm{~mm} \mathrm{Hg}$ due to moderate intracranial hypertension and moderate systemic hypotension. Heart rate appeared relatively unaffected during the time of terminal depolarization. Regions outlined by dashed black lines are shown in detail in Fig. 3, and the region outlined in solid gray is shown in Fig. 4. AC = alternating current.

ing. Spreading ischemia is a pathological vasoconstrictive response to depolarization observed in injured conditions, including hypoxia and impaired autoregulation. ${ }^{4,12}$
While hypoxia established the conditions for brain death, the pattern of repetitive SDs suggests that they also contributed causally to the dying process. For instance, 


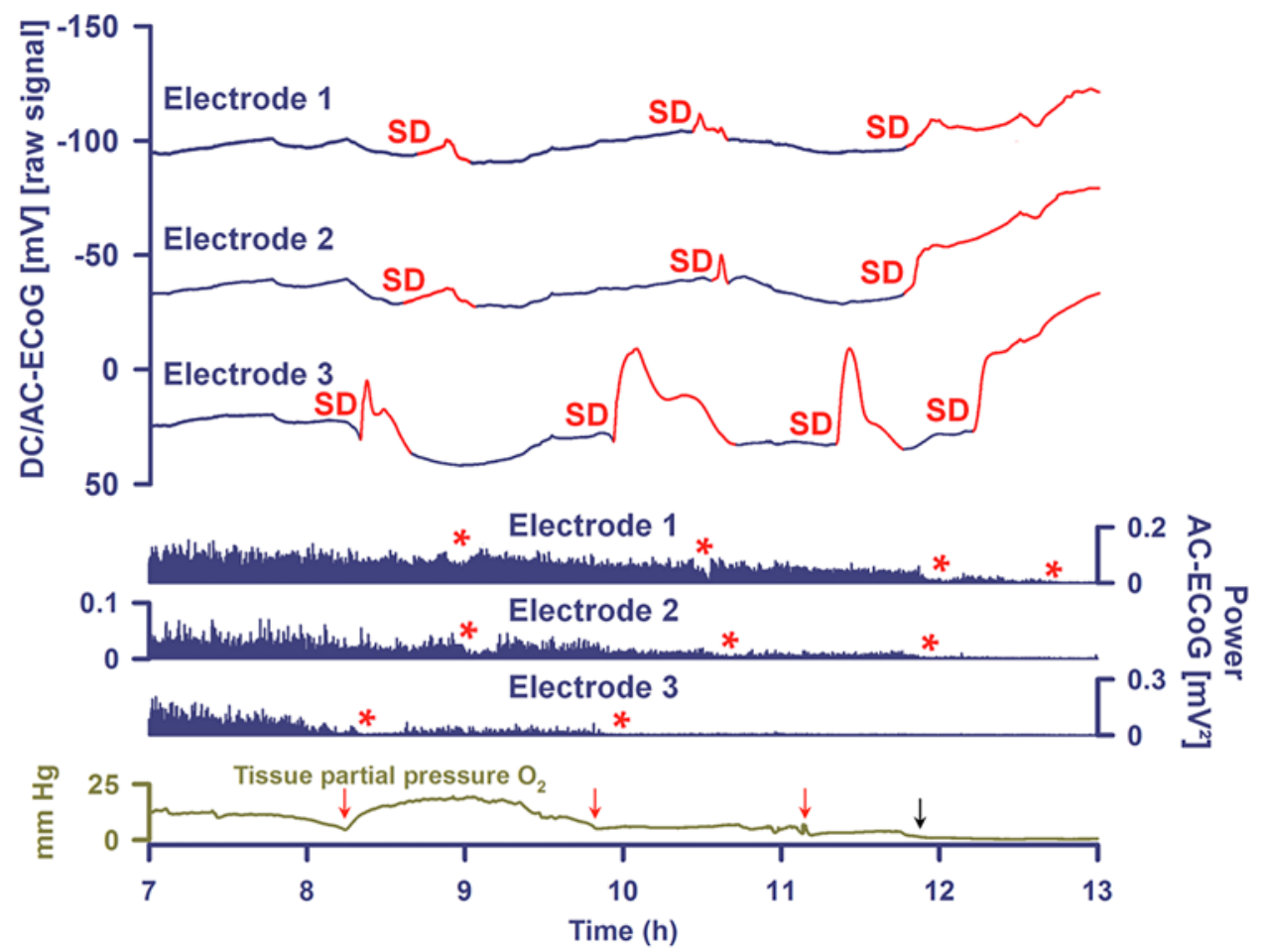

FIG. 3. Detailed course of brain oxygenation and SDs. A subset of the same traces in Fig. 2 are shown in greater detail. SDs are shown in red and depressions of spontaneous activity are labeled with asterisks. The expanded scale allows for better correlation of the initial drop in brain oxygenation $\left(\mathrm{p}_{\mathrm{ti}} \mathrm{O}_{2}\right)$ with each of the SDs (red arrows) and of complete brain hypoxic/ischemic damage with the final terminal depolarization.

focal brain isoelectricity (loss of spontaneous activity) developed in a spreading pattern with the local occurrence of SD and developed progressively and successively in adjacent tissue with subsequent, recurrent SDs. We also observed the progressive prolongation of depolarizations (e.g., electrode 3), ending eventually in the occurrence of persistent, terminal depolarization. The depolarization itself-measured by the DC shifts-reflects the breakdown of neuronal transmembrane ion gradients, which are necessary to generate synaptic potentials. In this case, the DC shifts became prolonged and eventually persistent, indicating the development of permanent neuronal damage. The same process is likely to have occurred throughout the brain as the final event leading to death. These observations are consistent with a vast body of preclinical data we have recently summarized on the role of hypoxia/ ischemia triggering $\mathrm{SD}$, the requirement for both hypoxia/ ischemia and SD for brain death or infarction to develop, and the strong evidence for a causal role of SD in this process. ${ }^{10}$

In another patient series, we have shown that terminal SD occurs in the cerebral cortex following activation of do-not-resuscitate/comfort-care orders in patients with severe brain injury. ${ }^{4}$ In such cases, extubation leads to cardiac failure and global hypoxia/ischemia with relatively abrupt onset. Isoelectricity of brain activity usually develops simultaneously across tissue (nonspreading depression of activity) as neurons hyperpolarize to reduce energy consumption as a final survival strategy. Terminal

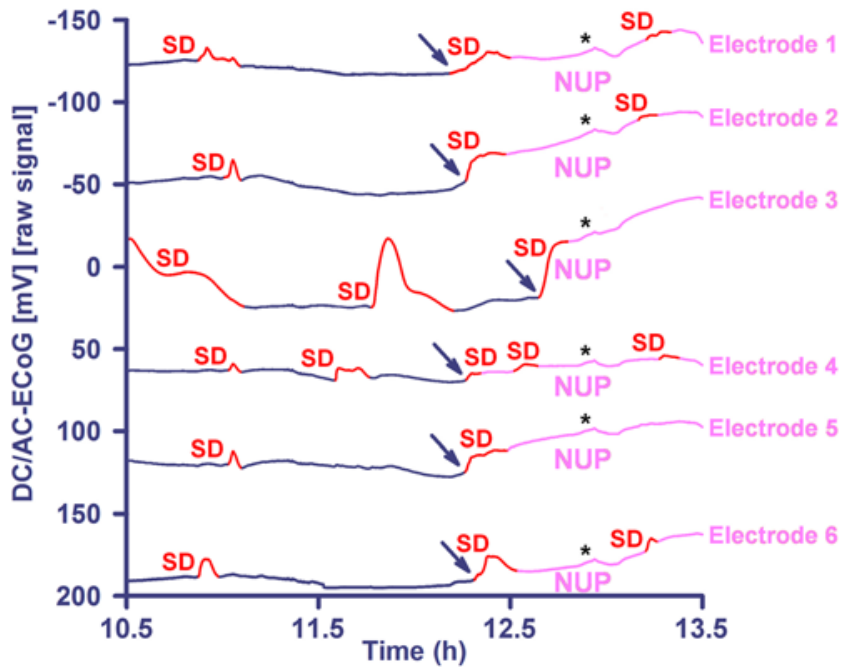

FIG. 4. Detail of terminal SDs and NUP. A subset of the same traces in Fig. 2 are shown in greater detail. SDs are shown in bright red and the negative ultraslow potential is denoted by pink. Blue arrows indicate the onset of terminal SD, which initiates simultaneously at electrodes 1,2 , 4 , and 5 , with subsequent spread to electrodes 6 and 3 . The terminal nature of this event is indicated by the persistence of the negative DC shift. This negative potential develops further through time as an NUP, which is thought to reflect the progressive development of tissue necrosis. Black asterisks indicate artifact due to the simultaneous occurrence across all channels. 
SD then occurs typically $2-5$ minutes later, when adenoside triphosphate supplies have been consumed. While the initial component of such terminal SDs was observed in 5 of 7 patients, the persistent nature of terminal depolarizations could not be proven due to confounding processes that also influenced recordings in this period.

The present case is distinct from this prior series and is remarkable in several respects. Most importantly, it is the first case to show that SDs contribute to progressive loss of brain function as measured by spontaneous brain activity in connection with, but preceding, clinical diagnosis of death, as well as being prior to and independent of major cardiopulmonary failure. Second, we have demonstrated terminal SD in the human brain by recording a persistent (> 60-minute) negative DC potential shift that spreads between adjacent electrodes. Finally, this case illustrates that SD can cause deterioration and ultimately death in tissue, at least focally if not also globally, even when the metabolism is initially relatively preserved. This is evidenced by the fact that spontaneous activity was still present at the start of the terminal cluster of SDs but that it progressively deteriorated to terminal isoelectric and depolarized states through the SD series. This contrasts with the pattern of nonspreading depression followed by terminal depolarization induced by the abrupt onset of severe ischemia. ${ }^{1,4}$ This sequence of events has not been previously observed because, until recently, it was not possible to measure this mechanism of brain death (which seems to occur ubiquitously in animal models of ische$\mathrm{mia}^{4,10}$ ) in human brain. SD cannot be reliably detected by noninvasive methods and even with the recent advances in SD monitoring, it is exceedingly rare to have ECoG recordings during the dying process, with the first such report in cases of cardiopulmonary collapse ${ }^{4}$ appearing this year.

SDs that are terminal or evolve into terminal depolarization while inducing persistent isoelectricity of the cerebral cortex have only been documented in a few instances in the literature. This was shown in rodents and cats in the context of delayed lesion growth after focal cerebral ischemia, ${ }^{13,16}$ in a swine with a declining mean arterial pressure at approximately $40 \mathrm{~mm} \mathrm{Hg},{ }^{11}$ in a patient with a large intracerebral hematoma, ${ }^{17}$ and in early and delayed cortical infarcts following aSAH. ${ }^{6,11}$ The present case is similar to these reports, but it is unique in demonstrating this expected pattern and mechanism in association with human clinical brain death using multiple physiological modalities, prior to circulatory collapse. Based on extensive experimental studies and these initial clinical observations, ${ }^{4,10}$ we suggest that SDs may be common if not ubiquitous in human brain death. Further study may lead to practical diagnostic and therapeutic applications.

\section{Acknowledgments}

Supported by grants from the Bundesministerium für Bildung und Forschung (BMBF) Center for Stroke Research Berlin (01 EO 0801), CENTER-TBI (FP7 no. 602150), and Deutsche Forschungsgemeinschaft (DFG DR 323/5-1) (J.P.D.).

Also supported by the University of New Mexico Department of Neurosurgery (A.P.C.) and NIH grants NS106901 and GM109089 (C.W.S.).

\section{References}

1. Dreier JP: The role of spreading depression, spreading depolarization and spreading ischemia in neurological disease. Nat Med 17:439-447, 2011

2. Dreier JP, Fabricius M, Ayata C, Sakowitz OW, Shuttleworth $\mathrm{CW}$, Dohmen C, et al: Recording, analysis, and interpretation of spreading depolarizations in neurointensive care: review and recommendations of the COSBID research group. $\mathbf{J}$ Cereb Blood Flow Metab 37:1595-1625, 2017

3. Dreier JP, Isele T, Reiffurth C, Offenhauser N, Kirov SA, Dahlem MA, et al: Is spreading depolarization characterized by an abrupt, massive release of Gibbs free energy from the human brain cortex? Neuroscientist 19:25-42, 2013

4. Dreier JP, Major S, Foreman B, Winkler MKL, Kang EJ, Milakara D, et al: Terminal spreading depolarization and electrical silence in death of human cerebral cortex. Ann Neurol 83:295-310, 2018

5. Dreier JP, Major S, Manning A, Woitzik J, Drenckhahn C, Steinbrink J, et al: Cortical spreading ischaemia is a novel process involved in ischaemic damage in patients with aneurysmal subarachnoid haemorrhage. Brain 132:1866-1881, 2009

6. Dreier JP, Woitzik J, Fabricius M, Bhatia R, Major S, Drenckhahn $\mathrm{C}$, et al: Delayed ischaemic neurological deficits after subarachnoid haemorrhage are associated with clusters of spreading depolarizations. Brain 129:3224-3237, 2006

7. Drenckhahn C, Windler C, Major S, Kang EJ, Scheel M, Vajkoczy P, et al: Complications in aneurysmal subarachnoid hemorrhage patients with and without subdural electrode strip for electrocorticography. J Clin Neurophysiol 33:250259, 2016

8. Hartings JA, Bullock MR, Okonkwo DO, Murray LS, Murray $\mathrm{GD}$, Fabricius $M$, et al: Spreading depolarisations and outcome after traumatic brain injury: a prospective observational study. Lancet Neurol 10:1058-1064, 2011

9. Hartings JA, Li C, Hinzman JM, Shuttleworth CW, Ernst GL, Dreier JP, et al: Direct current electrocorticography for clinical neuromonitoring of spreading depolarizations. J Cereb Blood Flow Metab 37:1857-1870, 2017

10. Hartings JA, Shuttleworth CW, Kirov SA, Ayata C, Hinzman JM, Foreman B, et al: The continuum of spreading depolarizations in acute cortical lesion development: examining Leão’s legacy. J Cereb Blood Flow Metab 37:1571-1594, 2017

11. Hartings JA, York J, Carroll CP, Hinzman JM, Mahoney E, Krueger B, et al: Subarachnoid blood acutely induces spreading depolarizations and early cortical infarction. Brain 140:2673-2690, 2017

12. Hinzman JM, Andaluz N, Shutter LA, Okonkwo DO, Pahl $\mathrm{C}$, Strong AJ, et al: Inverse neurovascular coupling to cortical spreading depolarizations in severe brain trauma. Brain 137:2960-2972, 2014

13. Hinzman JM, DiNapoli VA, Mahoney EJ, Gerhardt GA, Hartings JA: Spreading depolarizations mediate excitotoxicity in the development of acute cortical lesions. Exp Neurol 267:243-253, 2015

14. Lückl J, Lemale CL, Kola V, Horst V, Khojasteh U, OliveiraFerreira AI, et al: The negative ultraslow potential, electrophysiological correlate of infarction in the human cortex. Brain 141:1734-1752, 2018

15. Nakamura H, Strong AJ, Dohmen C, Sakowitz OW, Vollmar S, Sué M, et al: Spreading depolarizations cycle around and enlarge focal ischaemic brain lesions. Brain 133:1994-2006, 2010

16. Ohta K, Graf R, Rosner G, Heiss WD: Calcium ion transients in peri-infarct depolarizations may deteriorate ion homeostasis and expand infarction in focal cerebral ischemia in cats. Stroke 32:535-543, 2001

17. Oliveira-Ferreira AI, Milakara D, Alam M, Jorks D, Major 
S, Hartings JA, et al: Experimental and preliminary clinical evidence of an ischemic zone with prolonged negative DC shifts surrounded by a normally perfused tissue belt with persistent electrocorticographic depression. J Cereb Blood Flow Metab 30:1504-1519, 2010

18. von Bornstädt D, Houben T, Seidel JL, Zheng Y, Dilekoz E, Qin T, et al: Supply-demand mismatch transients in susceptible peri-infarct hot zones explain the origins of spreading injury depolarizations. Neuron 85:1117-1131, 2015

19. Woitzik J, Dreier JP, Hecht N, Fiss I, Sandow N, Major S, et al: Delayed cerebral ischemia and spreading depolarization in absence of angiographic vasospasm after subarachnoid hemorrhage. J Cereb Blood Flow Metab 32:203-212, 2012

\section{Disclosures}

Dr. Carlson reports being a consultant for Cerebroscope.

\section{Author Contributions}

Conception and design: Carlson. Acquisition of data: Carlson. Analysis and interpretation of data: Carlson, Major, Lemale, Dreier, Hartings. Drafting the article: Carlson, Shuttleworth, Hartings. Critically revising the article: Carlson, Shuttleworth, Dreier, Hartings. Reviewed submitted version of manuscript: Carlson, Shuttleworth, Dreier, Hartings. Approved the final version of the manuscript on behalf of all authors: Carlson. Study supervision: Carlson.

\section{Correspondence}

Andrew P. Carlson: University of New Mexico School of Medicine, Albuquerque, NM. andrewcarlson@salud.unm.edu. 